\title{
An Approach to Control the Positional Accuracy of Point Features in Volunteered Geographic Information Systems
}

\author{
Mennatallah H. Ibrahim ${ }^{1}$, Hesham A. Hefny ${ }^{3}$ \\ Computer Science, \\ Faculty of Graduate Studies for Statistical Research \\ Cairo University, Cairo, Egypt
}

\author{
Nagy Ramadan Darwish ${ }^{2}$ \\ Information Systems and Technology \\ Faculty of Graduate Studies for Statistical Research \\ Cairo University, Cairo, Egypt
}

\begin{abstract}
Volunteered geographic information (VGI) is a huge source of user-generated geographic information. There is an enormous potential to use VGI in different mapping activities due to its significant advantages. VGI is found to be richer and more up-to-date than authoritative geographic information. However, VGI quality is an obvious challenge that needs to be addressed in order to get the full potential of VGI. Positional accuracy is one of the important aspects of VGI quality. Although VGI positional accuracy can be high in some contexts, VGI datasets are characterized by a large spatial heterogeneity. This paper proposes an approach for controlling positional accuracy as well as decreasing the spatial heterogeneity of point features in VGI systems. A case study has been conducted in order to ensure the applicability and effectiveness of the proposed approach.
\end{abstract}

Keywords-Volunteered geographic information; quality control; positional accuracy; point features

\section{INTRODUCTION}

The proliferation of GPS enabled hand-held devices, the advances of communication technology, as well as, the development of Web 2.0 and collaborative mapping applications have dramatically extended the role of Geographic Information (GI) consumers. GI consumers are not only able to use and share GI, but also to create it as well. Consequently, a new phenomenon termed as volunteered geographic information has emerged. The term VGI has been coined by [1].

VGI is considered as an important source of user-generated geographic information. Nowadays, citizens who are not professional cartographer are able to participate in developing large and sophisticated cartographic projects. VGI not only creates new GI datasets, it can also enrich the traditional authoritative datasets as well. Furthermore, VGI is being studied as an alternative to authoritative data as it has been concluded that authoritative spatial datasets are often out-ofdate, incomplete, maintained expensively and their quality is inconsistent [2]. The author in [3] ensures that in some contexts, VGI can replace experts' data.

VGI has significant advantages that can overcome authoritative data limitations: (1) VGI can be accessed and obtained easily; (2) VGI is available over large areas either freely or at low cost; (3) VGI can be updated and maintained quickly by its users. Moreover, VGI is the only source of spatial data in certain regions due to security and/or financial issues [2].

As a result, there is an enormous potential to use VGI in different mapping activities and to integrate VGI with the authoritative datasets. However, VGI quality represents clear challenge to users who are concerned about quality assurance and quality validation of spatial data [4], [5]. Moreover, there are also many concerns about VGI quality that act as main barriers to integrate VGI with authoritative GI datasets [6].

As a result, VGI quality is an important research area that gains much interest [7]. One of the important aspects of VGI quality is the positional accuracy. As mentioned by [3], VGI with high quality is often associated with positional accuracy. VGI positional accuracy is characterized by a large spatial heterogeneity. The previous literature has shown little effort for controlling positional accuracy of volunteered point features in VGI systems. This paper proposes an approach to control positional accuracy of point features in VGI systems, in order to increase positional accuracy and decrease positional accuracy heterogeneity of such features across VGI datasets and maps within VGI systems.

The paper is structured as follows; it is divided into six sections. Section II presents an overview of VGI quality and positional accuracy. Section III presents the related work. Section IV introduces the proposed approach. Section V introduces the proposed algorithm. Section VI presents the conducted case study. Section VII, Shows the result of the case study. Finally, Section VIII summarizes the main points discussed in the paper.

\section{VGI QUALITY AND POSITIONAL ACCURACY}

Nowadays, private citizens provide online databases with vast amount of VGI through different websites and mobile applications. The provided VGI can be used in various disciplines as urban planning, public health, hydrology and disaster/risk management. As a result, VGI quality is critical especially if VGI will be used in spatial decision making processes. VGI quality is composite where it includes aspects dealing with data characteristics, volunteers' characteristics, and application context [8]. 
Generally, spatial data quality has eleven elements including lineage, positional accuracy, attribute accuracy, logical consistency, completeness, semantic accuracy and others [9]. Positional accuracy is one of the important elements of VGI quality. Positional accuracy is defined by [10] as the nearness of values that describe the position of a real-world entity in an appropriate coordinate system to the entity's true position in that system.

Generally, the main issues of VGI quality are the inconsistency of coverage and accuracy [2]. Although, VGI can reach in some contexts a high positional accuracy, a high spatial heterogeneity in positional accuracy has been indicated [12], [13]. As mentioned by [11], VGI is fundamentally imperfect with unknown and heterogeneous imperfection. From the reasons that lead to heterogeneity of VGI quality:

- Volunteers have different abilities, nature, expertise, motivation and priorities;

- Volunteers as well as VGI are not distributed equally over space;

- Volunteers may use different methods in VGI creation process (e.g. GPS and image tracing);

- Volunteers may use low accuracy devices in VGI creation process;

- There is no central coordination among volunteers;

- There are no systematic procedures for VGI creation or verification processes.

\section{RELATED WORK}

As mentioned before, inaccurate VGI may lead to significant implications. In [14], the authors concluded that quality control during VGI creation process is important. There are several previous studies that are concerned about analysis and assessment of VGI accuracy. Unfortunately, such studies give useful insights about VGI accuracy, but only assist indirectly in identifying an approach to control VGI accuracy.

The attempts to assess VGI quality have concluded that data heterogeneity is an intrinsic characteristic of VGI [15]. Fortunately, there are a number of earlier studies that are concerned about VGI quality control and assurance in general. Those previous studies can be categorized into three categories which are intrinsic, extrinsic and hybrid as shown in Table I. Intrinsic category includes studies that depend on internal factors for controlling and assuring VGI quality which are volunteers and/or volunteered data characteristics. Extrinsic category includes studies that depend on external factors such as gatekeepers and geographic contexts. Hybrid Category includes studies that rely on both internal and external factors together.

In [5], the authors propose an intrinsic approach that follows Linus's law [16]. The authors suggest that VGI holds an intrinsic quality assurance measure which is the number of contributors who works on a certain spatial unit. It is concluded that there is a positive relationship between VGI positional accuracy and number of volunteers; where beyond 15 volunteers within a square kilometer the positional accuracy becomes very good.

However, in [11], it is indicated that the practical implementation of the approach proposed in [5] can be challenging where, if the volunteered information is of poor quality data, increasing the number of volunteers may negatively affect VGI quality. Furthermore, in [17], it is indicated that Linus's law is more suitable for prominent geographic facts than for obscure ones. The authors in [11] also added that by following Linus's law, it is difficult to know how the single correct contribution can be chosen against the majority agreement of other volunteers.

In [17], the authors describe three VGI quality assurance approaches. First, the crowdsourcing approach which also relies on the assumption that the quality increase when more volunteers work on one area (considered as intrinsic approach). Second, the social approach which relies on a hierarchy of trusted individuals; act as gatekeepers to control VGI quality (considered as extrinsic approach). Third, the geographic approach which relies on geography knowledge to detect the reasonability of VGI (considered as extrinsic approach). Unfortunately, the approaches described in [17] cannot be adopted in all contexts as social approach is limited by the quality of the gatekeepers and the geographic approach is limited by the available contextual information [11].

In [11], the authors propose an intrinsic method based on latent class to characterize volunteers' quality relying on their contributions. The proposed method enables choosing only the appropriate volunteers for a specific VGI creation processes leading to highly accurate VGI. Such method filter volunteers before proceeding in VGI creation process leading to the possibility of losing volunteers who may help in the specified volunteering task because they are not classified as appropriate volunteers.

In [18], conceptual VGI quality assurance model for species occurrence observations is proposed. In the proposed model, VGI trustworthiness is a function of three contexts which are consistency with habitat, consistency with neighbors and volunteers' reputation where the level of trustworthiness is quantified by fuzzy set theory. The proposed model is considered to be hybrid as it depends on both internal factors (volunteer reputation) and external factors (consistency with habitat and consistency with neighbors). Unfortunately, the proposed model depends on extrinsic factors that may not be available all the time; moreover, such approach is appropriate only for certain contexts.

TABLE I. RELATED WORK CATEGORIZATION

\begin{tabular}{|l|c|c|c|}
\hline \multirow{2}{*}{ Study } & \multicolumn{3}{|l|}{ Category } \\
\hline & Intrinsic & Extrinsic & Hybrid \\
\hline$[5]$ & $\checkmark$ & & \\
\hline$[17]$ - Crowd-sourcing Approach & $\checkmark$ & & \\
\hline$[17]$ - Social Approach & & $\checkmark$ & \\
\hline$[17]-$ Geographic Approach & & $\checkmark$ & \\
\hline$[11]$ & $\checkmark$ & & \\
\hline$[18]$ & & & $\checkmark$ \\
\hline
\end{tabular}


As mentioned by [2], the issue of VGI quality is a trending research topic because of the vast amount of data and the inapplicability of Linus's law. The previous related work was concerned about controlling and assuring VGI quality with almost no efforts directed specifically to control positional accuracy of volunteered point features in VGI systems. The aim of this paper is to propose an approach that controls the positional accuracy of volunteered point features in VGI systems.

\section{PROPOSED APPROACH}

The proposed approach aims to controls the positional accuracy of point features in VGI systems in order to increase the positional accuracy and decrease the spatial heterogeneity of such features; it helps to creates highly accurate and consistent volunteered geographic maps and datasets that contain no outliers. The proposed approach aims also to overcome the limitations of the previous related work. The proposed approach is hybrid where it depends on both internal and external factors for controlling VGI positional accuracy.

First, as shown in Fig. 1, we characterized the positional accuracy of the volunteered point features through three main dimensions which are volunteers, contributions and content. Each dimension is concerned about number of elements as follows:

- Volunteer. Concerned about the volunteers themselves; whether the volunteer is trained or not, volunteer's local knowledge and volunteer's location during his/her contribution.

- Content. Concerned about the volunteered point (VP); volunteers' density which reflects the number of volunteers in a specific area, volunteers' agreement which reflects the agreement of volunteers on a specific VP and the geographic context of the VP.

- System Design. Concerned about the environment through which the volunteers perform their volunteering task; task definition, the instructions given to the volunteers in order to accomplish their tasks accurately, the user interface through which the volunteers provide their VPs.

\section{A. Volunteer Dimension}

The first dimension to characterize the positional accuracy of VPs is the volunteer dimension. Volunteer dimension describes three aspects concerning the volunteers themselves which are training, local knowledge and location.

1) Training: Training is one of the important aspects that affect VGI quality [19], [8]. With appropriate training, volunteers are able to improve more than domain's expert [20]. Contributions from volunteers who have no formal geographic training may lead to inaccurate VGI [21]. Even remote volunteers when they get trained their contribution errors could be minimized [22]. In the proposed approach, all citizens will be allowed to volunteer; however, volunteers who got a training or had a training material will be tagged as trained volunteers.

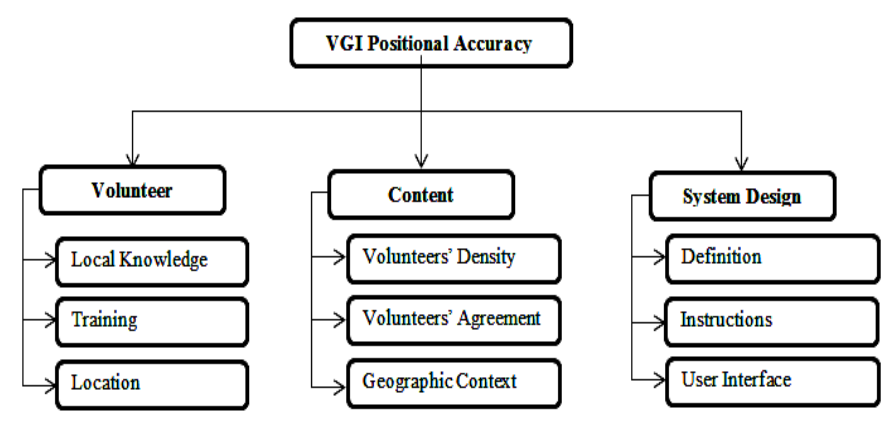

Fig. 1. Characterization of Positional Accuracy of Volunteered Point Features.

2) Local knowledge: One of VGI quality indicators is the volunteers' local knowledge [23]. Local Knowledge can lead to highly accurate and up-to-date local maps which sometimes provide information with quality higher than the experts' maps [20], [8], [17]. Volunteers are more familiar with their own area than distant authoritative experts, so volunteers with their local knowledge could create a map of their area more effectively. In the proposed approach, the volunteers are asked to determine their residence place before proceeding in VGI creation process. We assumed that the volunteers' local knowledge is related to their residence city and region.

3) Location: We have observed through our case study that if a point feature is located inside a building, only the volunteers who are located in the same area of the VP are able to determine the VP location accurately. Consequently, our proposed approach will automatically collect metadata about the volunteers' location during performing their volunteering task. If the VP is inside a building, the distance between the volunteer and the VP will be calculated using Euclidean distance. The volunteer has to be at a distance of 100 meters or less from his/her VP.

In the proposed approach, the volunteers are classified according to their local knowledge and training skills (i.e. whether they got trained or not). Volunteer are assigned a weight according to their class, as shown in Fig. 2. The proposed approach classifies volunteers according to their local knowledge with respect to their created VGI as follows:

- Local volunteers. Volunteering in their residence city (called city volunteer) or in their residence regions (called region volunteer). Local volunteers will be assigned a Weight ${ }_{1}\left(\mathrm{~W}_{1}\right)$ up to 1 , where $\mathrm{W}_{1}=1$, if cityvolunteer or $\mathrm{W}_{1}=0.75$, if region-volunteer.

- Remote volunteers. Volunteering in their residence country but not their residence region (called country volunteers) or in other country (called other-country volunteers). Remote volunteers will be assigned $\mathrm{W}_{1}$ up to 0.35 . $\mathrm{W}_{1}=0.35$, if country-volunteers or $\mathrm{W}_{1}=0$, if other-country-volunteers.

All volunteers except city-volunteers are further classified into trained or not trained volunteers and assigned Weight ${ }_{2}$ $\left(\mathrm{W}_{2}\right)$. For trained volunteers, $\mathrm{W}_{2}=0.25$ and for untrained volunteers, $\mathrm{W}_{2}=0$. 


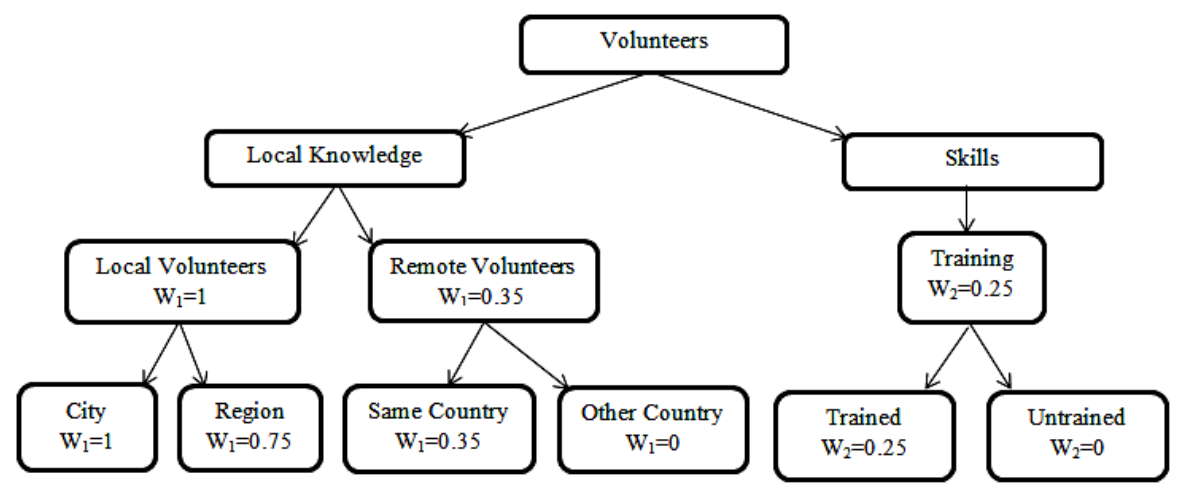

Fig. 2. Volunteers' Classification According to their Local Knowledge and Training.

Consequently, we have seven types of volunteers which are city, region-trained, region-untrained, country-trained, countryuntrained, other-country-trained, and other-country-untrained. Each volunteers' type is assigned a Volunteer's Weight (VW) which is the summation of $\mathrm{W}_{1}$ and $\mathrm{W}_{2}$ (as shown in Table II).

The highest weight is assigned to city-volunteers due to their high local knowledge and also to region-trained volunteers; VW for both will be 1 . Followed by region-trained volunteers $(\mathrm{VW}=0.75)$. Then $\mathrm{VW}$ decreases for country volunteers as the local knowledge of the volunteers respecting other regions and cities decreases $(\mathrm{VW}=0.35)$ and if country volunteer is trained VW will be increased to 0.55 . Othercountry volunteers are assigned the lowest weight if they are trained VW will be 0.25 and zero if they are not trained.

\section{B. Content Dimension}

The second dimension is content dimension which is concerned about the characteristics of the VPs. Firstly, the volunteers' density which reflects the number of volunteers who have volunteered within a specific area around the VP. Secondly, the volunteers' agreement that indicates whether there is an agreement on a specific VP or not. Thirdly, the geographic context is concerned about the suitable geographic content of the VPs. The VP will be assigned a weight according to the satisfied characteristics as shown in Fig. 3.

1) Volunteers' density: In [5], it is concluded that there is a positive relationship between VGI positional accuracy and number of volunteers. [24] and [25] also ensures that positional accuracy positively correlate with number of volunteers. In our approach the number of volunteers who volunteered within a distance of one kilometer around each VP is counted. If the number of volunteers exceeded 15 , the volunteered point will be assigned a weight of 0.30 .

2) Volunteers' agreement: Majority agreement provides confidence in the VP. According to standard consensus-based approach, if contributions from multiple volunteers are available, the majority view is followed, unfortunately, such approach may cause the one in million correctly volunteered information to be lost [11]. In our proposed approach, agreement on a specific VP is considered without losing the unique VP that has no agreement by assigning weight, instead of excluding VPs. A buffer zone of radius 15 meters is created around each VP. The number of volunteers within the created buffer is counted; indicating the number of volunteers who agreed that there is a true point in the specifoed location. If the number of volunteers is two or more, the volunteered point will be assigned 0.40 for its weight.

3) Geographic context: According to Tobler's first law in [26], a location is likely to be more similar to its surrounding area than distant area. In other word, Tobler's law suggests that information about a location should be consistent with what is already known about its surrounding area [17]. As mentioned by [12], VGI quality can be enhanced by studying the geographic context of the map's objects, however little work has been done using such an approach. Our proposed approach will consider the VPs' geographic context. Two kinds of geographic context rules are allowed to be set before VGI creation process for including and excluding VPs. Any volunteered point that violates any of the exclusion rules will be excluded. On the other hand, any volunteered point that meets the inclusion rules will be assigned 0.30 for its weight.

The VP is assigned Content Weight $(\mathrm{CW})$ as shown in Table III, in case that a VP satisfied all aspects of content dimension, $\mathrm{CW}=1$. If the VP satisfied the volunteers' density aspect with any other aspect, the $\mathrm{CW}=0.70$. If the VP satisfied the volunteers' density and geographic contexts aspects, $\mathrm{CW}=0.60$. If none of the aspects are satisfied the $\mathrm{CW}$ will be equal to zero.

\section{System Design Dimension}

Important aspects that affect VGI quality are the definition provided to volunteers about their volunteering tasks, instructions of how to create the targeted VGI accurately and the interface through which volunteers perform their volunteering tasks.

TABLE II. WEIGHT OF DIFFERENT VOLUNTEERS' TYPES

\begin{tabular}{|l|l|}
\hline Volunteer's Type & VW \\
\hline City & 1 \\
\hline Region-Trained & 1 \\
\hline Region-Untrained & 0.75 \\
\hline Country-Trained & 0.55 \\
\hline Country-Untrained & 0.35 \\
\hline Other-Country-Trained & 0.25 \\
\hline Other-Country-Untrained & 0 \\
\hline
\end{tabular}




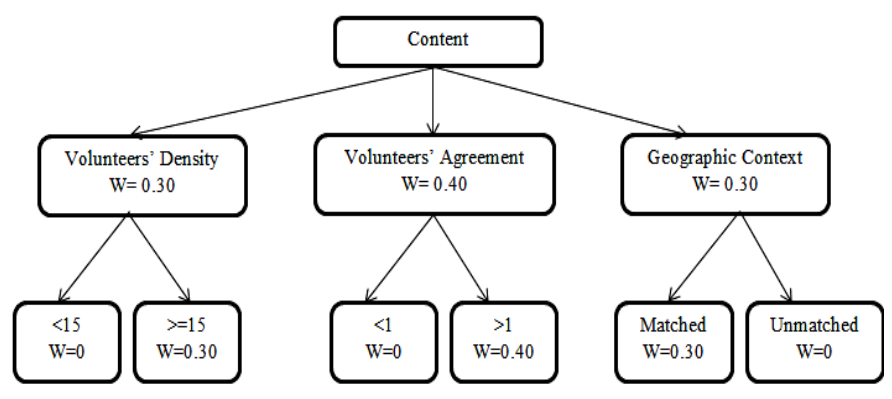

Fig. 3. Volunteered Points' Weight.

TABLE III. WEIGHT ASSIGNED TO VPS

\begin{tabular}{|l|l|}
\hline Content Dimension Satisfied Aspects & Weight \\
\hline All aspects & 1 \\
\hline Volunteers' Density and Volunteers' Agreement & 0.70 \\
\hline Volunteers' Density and Geographic Context & 0.60 \\
\hline Volunteers' Agreement & 0.40 \\
\hline Volunteers' Density or Geographic Context & 0.30 \\
\hline None of the aspects & 0 \\
\hline
\end{tabular}

1) Definition and instructions: In order to avoid confusing volunteers, before proceeding in and also during VGI creation process: (1) the task, its purpose and it's nature is defined and explained in a clear statement to all volunteers; (2) instructions about how to perform the volunteering task is provided to volunteers; and (3) the task's requirements and constraints is clearly specified.

2) User interface: A simple well-designed user interface is adopted by the proposed approach. Such design makes the VGI system clear and easy to use in order to avoid demotivating the targeted volunteers and to help them to perform their volunteering tasks accurately.

\section{PROPOSED AlgorithM}

Once a volunteer inserted a VP's location on the map, the volunteer is assigned a weight according to Table II and the VP is checked against geographic context exclusion rules. If the VP violates any of the exclusion rules, it will be excluded from the VGI dataset. Otherwise, the VP will be checked if it is located inside a building or not. If the VP is located in a building, it will be included in the VGI dataset only if its volunteer is located at a distance of 100 meter or less from it. The VP is then assigned a weight according to Table III.

Volunteer's and VP's weight are then checked against each other, as shown in Table IV. The volunteers with VW equals to 1 , all his/her VPs will be accepted. If VW equals to 0.75, only VPs with CW equals to $(1,0.7,0.6,0.4$ or 0.3$)$ will be accepted. If the VW equals to 0.55 , only VPs with $\mathrm{CW}$ equals to $(1,0.7,0.6$ or 0.4$)$ will be accepted. If VW equals to 0.35 , only VPs with $\mathrm{CW}$ equals to $(1,0.7$ or 0.6$)$ will be accepted. If VW equals 0.25 , only VP with CW equals to ( 1 or 0.7 ) will be accepted. Finally, if VW equals to 0 , only VP with $\mathrm{CW}$ equals to 1 will be accepted. VP will be included in VGI dataset only if it follows Table IV, otherwise, it will be excluded.
As mentioned before, each VP is assigned two weights which are VW and CW. VW reflects the local knowledge and training skills of the volunteer, while $\mathrm{CW}$ reflects the volunteers' density, volunteers' agreement and if inclusion geographic context rule has been met.

The locations of all VPs which gained the weight of volunteers' agreement will be replaced by an Estimated Volunteered Point's latitude and longitude (EVP $(x, y))$. The EVP (x,y) is calculated as the center of area, as shown in Fig. 4, based on a computed generalized weight (wi) as follows:

$\operatorname{EVP}(\overline{\mathrm{x}})=\mathrm{VP}$ latitude $\mathrm{x}=\sum_{i=1}^{n} \mathrm{VP}\left(\mathrm{y}_{\mathrm{i}}\right) \mathrm{w}_{\mathrm{i}} / \sum_{i=1}^{n} \mathrm{w}_{\mathrm{i}}$

$\operatorname{EVP}(\mathrm{y})=\mathrm{VP}$ longitude $\mathrm{y}=\sum_{i=1}^{n} \mathrm{VP}\left(\mathrm{y}_{\mathrm{i}}\right) \mathrm{w}_{\mathrm{i}} / \sum_{i=1}^{n} \mathrm{w}_{\mathrm{i}}$

$\mathrm{w}_{\mathrm{i}}=\left(V W_{i}+P W_{i}+N W_{i}\right) / 3$

Where NWi is the normalized number of VPs around each VPi within a buffer zone of 5 meters.

$\mathrm{NW}_{\mathrm{i}}=\frac{\text { Number of VPs around VPi within } 5 \mathrm{~m}}{\text { Number of VPs around VPi within } 15 \mathrm{~m}}$

TABLE IV. VOLUNTEER WEIGHT AND CONTENT WEIGHT JOINING

\begin{tabular}{|l|l|l|l|l|l|l|}
\hline VW & \multicolumn{6}{l}{ CW } \\
\hline & $\boldsymbol{1}$ & $\mathbf{0 . 7 0}$ & $\mathbf{0 . 6 0}$ & $\mathbf{0 . 4 0}$ & $\mathbf{0 . 3 0}$ & $\mathbf{0}$ \\
\hline $\boldsymbol{1}$ & yes & yes & yes & Yes & yes & yes \\
\hline $\mathbf{0 . 7 5}$ & yes & yes & yes & Yes & yes & x \\
\hline $\mathbf{0 . 5 5}$ & yes & yes & yes & Yes & x & x \\
\hline $\mathbf{0 . 3 5}$ & yes & yes & yes & X & x & x \\
\hline $\mathbf{0 . 2 5}$ & yes & yes & x & X & x & x \\
\hline $\mathbf{0}$ & yes & x & x & X & x & x \\
\hline
\end{tabular}

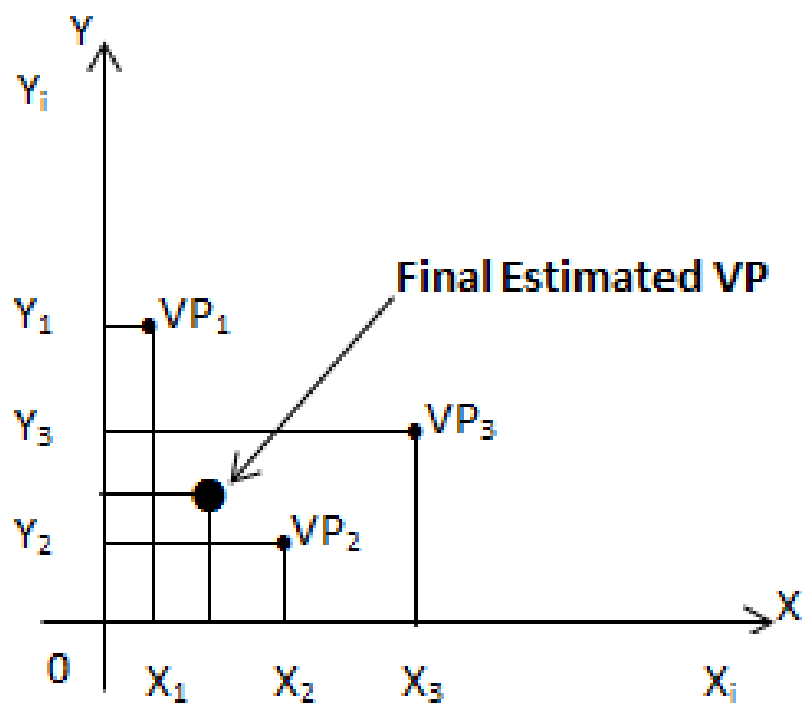

Fig. 4. Estimated Volunteered Point's Location in Case of Agreement. 
The steps of the proposed algorithm are listed as follows:

\begin{tabular}{|c|}
\hline $\begin{array}{l}\text { 1. Volunteer inserts a VP's location on the map } \\
\text { 2. Volunteer is assigned a weight (VW) according to Table II } \\
\text { 3. IF (VP violates any geographic context rule) THEN } \\
\text { 4. } \quad \text { Exclude VP from VGI dataset } \\
\text { 5. END IF } \\
\text { 6. IF (VP is located inside a building) AND (Volunteer is within a } \\
\text { distance of } 100 \mathrm{~m} \text { or less from the VP) THEN } \\
\text { 7. Include VP in VGI dataset } \\
\text { 8. ELSE } \\
\text { 9. } \\
\text { 10. END IF } \\
\text { 11. VP is assigned a weight (PW) according to Table III } \\
\text { 12. IF (VW and PW follow Table IV) THEN } \\
\text { 13. Include VP in VGI dataset } \\
\text { 14. ELSE } \\
\text { 15. Exclude VP from VGI dataset } \\
\text { 16. END IF } \\
\text { 17. IF (there is volunteers' agreement on a certain point feature) } \\
\text { 18. FOR each agreed VPi } \\
\text { 19. Compute NWi according to (4) } \\
\text { 20. Compute wi according to (3) } \\
\text { 21. END FOR } \\
\text { 22. END IF } \\
\text { 23. Compute EVP ( } x \text { ) according to (1) } \\
\text { 24. Compute EVP ( } y \text { ) according to (2) } \\
\text { 25. Replace all agreed VPs' longitude and latitude with EVP( } \mathrm{x}, \mathrm{y} \text { ) } \\
\text { in VGI dataset }\end{array}$ \\
\hline
\end{tabular}

\section{CASE StUdy}

In order to prove the feasibility and the applicability of the proposed approach a case study has been conducted. A VGI mobile application that adopts the proposed approach and algorithm has been developed and published on Google play store, see Fig. 5. The aim of the developed application is to collect volunteered data about the locations of automated teller machines (ATMs) for a specific bank in Cairo, Egypt. The volunteers were asked to locate the ATMs on the application's map. The developed application has a user friendly interface which is clear, simple and easy to use. Before proceeding in their tasks, the volunteers were provided detailed information about the aim and the purpose of the application. A detailed instructions before and during using application were available to volunteers.

First, we identified rules for the geographic context of the targeted point features (ATMs) as follows:

\section{A. Exclusion Rules}

- Rule 1: The ATMs are within Cairo boundaries.

- Rule 2: The ATMs is not within the location of any other banks' buildings.

- Rule 3: The ATMs is not within the location of other bank's ATM.
- Rule 4: The ATMs is not within any water surfaces (e.g. rivers, and seas).

\section{B. Inclusion Rules}

- Rule 5: The ATMs are within the location of the specified bank.

- Rule 6: The ATMs are within area that is categorized as a business area (e.g. shops centers).

The volunteers were then invited to register to the application and while registering they were asked to indicate their residence location. Before proceeding to contribute, an instruction page was displayed to the volunteer in order to identify exactly the volunteering task and it's purposes and also to indicate the requirements needed for that task (to turn on GPS and Wi-Fi).

Once registered, the volunteers were able to contribute by adding the location of the ATMs that they know or deal with on the map. A volunteer was also able to view, edit and delete other volunteers' contributions. Each function that can be performed on the application (contribute, view, edit or delete) has an instruction page to guide the volunteers how to perform it accurately. While volunteering the volunteer's position was caught and saved in the application's database. We had around 300 VPs from 45 volunteers. Ten volunteers have been trained before using the application on how to use the application efficiently, how to locate ATMs on the map accurately, and some illustrative examples were shown to them.

\section{RESULTS AND DISCUSSION}

We compared the VPs' dataset with a reference dataset that contain the real positions of the investigated point feature (ATMs). First, a buffer zone of radius 50 meters was created around each VP in the VGI dataset. Second, the distance between the VP and ATMs in the reference dataset within the created buffer, is measured using Euclidean distance and the nearest ATM is detected as a matched point. We evaluated the spatial errors for each VP and matched ATM, see Table V.

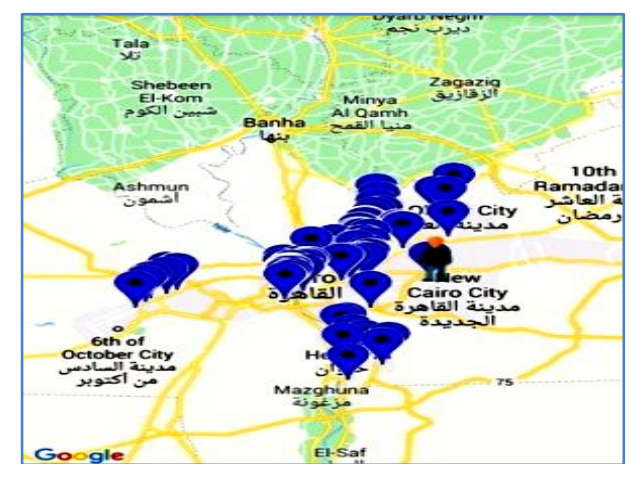

Fig. 5. Part of VPs on Application's Map.

TABLE V. SPATIAL ERrors Statistics OF VolunteERED ATMs

\begin{tabular}{|l|l|l|l|l|}
\hline \multicolumn{5}{|l|}{ Spatial Error (m) } \\
\hline Min. & Max. & Mean & $\begin{array}{l}\text { Standard } \\
\text { Deviation }\end{array}$ & $\begin{array}{l}\text { Coefficient of } \\
\text { Variation }\end{array}$ \\
\hline 0 & 5.761835 & 2.410364 & 1.336997 & $55.46868 \%$ \\
\hline
\end{tabular}


The minimum spatial error is zero which means that ATM's position has been located accurately. The maximum spatial error is 5.761835; indicating that our VPs' data set has no outliers. The mean of spatial errors is 2.410364; by considering the average accuracy of GPS devices (6-10 meters), all VPs' location can be considered as highly accurate. Finally, the standard deviation is 1.336997 and coefficient of variation is $55.46868 \%$.

We compared our results with the results of a previous study that aimed to assess the positional accuracy of point features in other VGI systems which is OpenStreetMap [14], the coefficient of variation shows that our approach has significantly decreased the spatial heterogeneity of the final VPs' dataset and the mean shows that our proposed approach increased the positional accuracy of VGI point features.

Unlike previous related work, our proposed approach is concerned specifically about controlling positional accuracy of point features; it increases positional accuracy and decreases spatial heterogeneity of such features. Furthermore, our proposed approach overcomes the limitations of the previous related work: (1) the proposed approach does not mainly depend on linus law, where it is concerned also about volunteers' quality and geographic context; (2) It does not depend on an external factor as a gatekeeper or a moderator; (3) It provides the flexibility of adding any suitable and available geographic contexts rules to get the benefit of using geographical knowledge to detect the reasonability of VGI; (4) It provides also the flexibility to deal with various types of volunteers regardless their experience or reputation without excluding any volunteers' contribution; (5) the proposed approach can be adapted to be suitable for various contexts.

\section{CONCLUSIONS}

VGI quality is an obvious challenge that limits VGI usage and integration with authoritative datasets. The previous literature primarily focuses on assessing and evaluating VGI quality with few efforts concerned about VGI quality assurance and control. The previous literature lacks effort to control positional accuracy of point features in specific. In order to fill this gap an approach to control the positional accuracy of VGI has been proposed and a case study has been conducted to prove the applicability and effectiveness of the proposed approach.

\section{REFERENCES}

[1] M. Goodchild, "Citizens as sensors: the world of volunteered geography," GeoJournal, vol. 69, pp. 211-221. 2007.

[2] H. Zhang, and J. Malczewski, "Quality evaluation of volunteered geographic information: The case of OpenStreetMap," Volunteered Geographic Information and the Future of Geospatial Data, pp. 19-46, 2017.

[3] J. Meier, "An analysis of quality for volunteered geographic information," thesis, Wilfrid Laurier University, Waterloo, Canada, 2015.

[4] M. Eshghi, and A. Alesheikh, "Assessment of completeness and positional accuracy of linear features in Volunteered Geographic Information (VGI)," in International Conference on Sensors \& Models in Remote Sensing \& Photogrammetry, 2015, p. 169.
[5] M. Haklay, S. Basiouka, V. Antoniou and A. Ather. "How many volunteers does it take to map an area well? The validity of Linus' law to volunteered geographic information," The Cartographic Journal, vol. 47, pp. 315-322. 2010.

[6] A. Flanagin, and M. Metzger, "The credibility of volunteered geographic information," GeoJournal. vol. 72, pp. 137-148. 2008.

[7] L. See, et al. "Crowdsourcing, citizen science or volunteered geographic information? The current state of crowdsourced geographic information," ISPRS International Journal of Geo-Information, vol. 5 .2016 .

[8] L. Criscuolo, et al., Handling quality in crowdsourced geographic information. European Handbook of Crowdsourced Geographic Information. London: Ubiquity Press,2016.

[9] P. Van Oort, "Spatial data quality: from description to application", thesis, Wageningen University, Wageningen, Netherlands. 2006.

[10] J. Drummond, J. "Positional accuracy," Elements of spatial data quality, pp. 31-58. 1995.

[11] G. Foody, et al. "Accurate attribute mapping from volunteered geographic information: issues of volunteer quantity and quality," The Cartographic Journa, vol. 52, pp. 336-344. 2015.

[12] A. Vandecasteele, and R. Devillers, "Improving volunteered geographic information quality using a tag recommender system: The case of OpenStreetMap," OpenStreetMap in GIScience, pp. 59-80, 2015.

[13] M. Haklay, "How good is volunteered geographical information? A comparative study of OpenStreetMap and Ordnance Survey datasets," Environment and planning B: Planning and design, vol. 37, pp. 682-703. 2010.

[14] S. Jackson, et al. "Assessing completeness and spatial error of features in volunteered geographic information," ISPRS International Journal of Geo-Information, vol. 2, pp. 507-530. 2013.

[15] D. Bégin, R. Devillers and S. Roche, "Assessing Volunteered Geographic Information VGI quality based on contributors' mapping behaviours," in Int. Arch. Photogramm. Remote Sens. Spat. Inf. Sci, 2013, p. 149.

[16] E. Raymond, "The cathedral and the bazaar," Knowledge,Technology \& Policy, vol. 12, pp. 23-49. 1999.

[17] M. Goodchild, and L. Li, "Assuring the quality of volunteered geographic information," Spatial statistics, vol. 1, pp. 110-120. 2012.

[18] H. Vahidi, B. Klinkenberg,, and W. Yan, "A fuzzy system for quality assurance of crowdsourced wildlife observation geodata," In IEEE 2017 International Electronics Symposium on Knowledge Creation and Intelligent Computing (IES-KCIC), 2017, P. 55.

[19] S. Ghosh, et al. "Crowdsourcing for rapid damage assessment: The global earth observation catastrophe assessment network (GEO-CAN)," Earthquake Spectra, vol. 27, pp. 179-198. 2011.

[20] L. See, et al. "Comparing the quality of crowdsourced data contributed by expert and non-experts," PloS one, vol. 8. 2013.

[21] M. Schwind., K. Davis, and P. Baldridge. "Analyzing volunteer geographic information accuracy and determining its capabilities for scientific research data," Honors and Undergraduate Research. 2014. Available electronically from http : / /hdl .handle .net /1969 .1 /152055.

[22] M. Eckle, and P. de Albuquerque, "Quality assessment of remote mapping in OpenStreetMap for disaster management purposes," in ISCRAM conference. 2015.

[23] M. Van Exel, E. Dias, and S. Fruijtier, "The impact of crowdsourcing on spatial data quality indicators," in Proc. of the GIScience, 2010, p.14.

[24] V. Antoniu, "Volunteered geographic information measuring quality, understanding the value," GEOmedia, vol. 20, pp. 38-45, 2016.

[25] P. Neis, D. Zielstra and A. Zipf, "Comparison of volunteered geographic information data contributions and community development for selected world regions," Future Internet, vol. 5, pp. 282-300. 2013.

[26] H. Miller, "Tobler's first law and spatial analysis," Annals of the Association of American Geographers, vol. 94, pp. 284-289. 2004. 